\title{
EXPERIMENTOS CON EL MODELO MEXICANO DE INSUMO-PRODUCTO
}

\author{
PEDRO URIBE ${ }^{1}$
}

\section{INTRODUCCIÓN}

El PROPósito de este trabajo es comentar sobre algunos resultados empíricos obtenidos de la aplicación del método RAS de Stone et al. al modelo mexicano de isumo-producto. No teniendo mucha evidencia en la cual basarse para la comprobación de las matrices ajustadas vía RAS versus la realidad, se ha tratado de analizar la consistencia de los resultados y la plausibilidad empírica de las conclusiones derivadas de nuestra serie de matrices. Otra posibilidad, por explorar aún, es utilizar otros métodos de ajuste, tales como el enfoque de programación lineal de Matuszewski et al. [1964].

En la sección II se discute el ras a la luz del teorema de no-sustitución de Klein [1952], de acuerdo al cual los coeficientes del RAS son razones de cambio de los precios en el tiempo: ésto se sujetará a prueba empírica en la sección IV. En la sección III se describen algunos pasos utilizados para la estimación de la serie de matrices. En la IV se describe una prueba de corto plazo para la predicción de marginales futuros a partir de una matriz conocida y multiplicadores constantes del RAS. Se concluye que, aunque los errores crecen linealmente con el tiempo, unos cuantos sectores explican casi toda su magnitud, de tal manera que la extrapolación puede ser segura en el corto plazo, si se sigue la huella de algunos sectores clave.

La sección $\mathrm{V}$ trata de la predicción de la demanda intermedia, dadas la demanda final y la matriz de coeficientes. La edad de la matriz de cieficientes se vuelve crucial, siendo mejor la extrapolación proporcional a la demanda final que la basada en insumo-producto cuando la edad de la matriz excede a 10 años. En la sección VI se estudia el efecto de los cambios de coeficientes sobre la demanda intermedia. Se obtiene que, por un lado, los cambios de coeficientes - minimizados en promedio por

1 El autor está en deuda con Gerardo Bueno por el continuo apoyo en esta investigación. Se ha beneficiado mucho de los comentarios de Paul Zarembka sobre una versión anterior y agradece los comentarios del Prof. Phoebus Dhrymes sobre la presentación oral en la Conferencia de la Ciudad de México (1974). Desde luego, ninguno de ellos es responsable por los errores y mal empleo de conceptos que contenga este trabajo. Deseo también agradecer a la Oficina de Computación de Petróleos Mexicanos por el libre uso de la terminal, tiempo de computación y archivos en discos. Traducción de Ana Catalina Mayoral, revisada por el autor. 
el RAS - son determinantes en extremo importantes de los cambios en la demanda intermedia de la economía mexicana. Por otro lado, los resultados son empíricamente plausibles en el sentido de que indican un proceso de sustitución que ocurre en la economía, en el que los insumos "tradicionales" (bienes agrícolas, minerales) son sustituidos por productos químicos; una gran proporción del crecimiento del llamado sector "moderno" se explica por cambios en los coeficientes.

La sección VII supone que los coeficientes son generados por una función de producción Cobb-Douglas, a través de la maximización de ganancias, dados precios exógenos. Parece ser que los movimientos de los precios han sido tan pequeños que ningún cambio significativo se predice por el modelo Cobb-Douglas; por lo tanto, los cambios en los coeficientes son principalmente el resultado de un cambio tecnológico ex-ante. El cálculo del cambio ex-ante en el modelo Cobb-Douglas lleva prácticamente a los mismos resultados observados en la sección VI, con una elevada plausibilidad empírica.

Por último, en la sección VIII se sugiere un modelo de cambio tecnológico, libre de función producción, cuya comprobación empírica está todavía en investigación.

\section{SOBRE EL RAS Y SU INTERPRETACIÓN}

Aunque el método RAS para actualizar tablas de insumo-producto es conocido, conviene mencionar brevemente algunas de sus características principales que serán relevantes en la secuencia. Sean $A$ y $B$ dos matrices no negativas cuadradas del mismo orden, tales que para ambas $A$ y $B$, ninguno de sus renglones o columnas consiste enteramente de ceros.

$B$ es una transformada RAS de $A$ si existen matrices diagonales estrictamente positivas $r$ y $s$ tales que $B=r A s$. Claramente, la relación " $B$ es una transformada RAS de $A$ " es una relación de equivalencia. ${ }^{2}$

La transformada RAS de una matriz de coeficientes de insumo-producto en $t, A(t)$ fue propuesta originalmente por Leontief como un modelo para la matriz de coeficientes $A(t+1)$. El profesor Stone y su grupo en Cambridge, Inglaterra; (véase Stone et al. [1963], propusieron un método para encontrar una transformada RAs específica: aquélla en que $r A(t) s$ sume los totales marginales conocidos para $(t+1)$. Las transformadas RAS han sido extensamente estudiadas por M. Bacharach, anteriormente miembro del grupo de Stone (véase Bacharach [1970]). Bacharach demuestra que, si $A$ y $B$ se miden en unidades tales que $\Sigma_{i j} a_{i j}=\Sigma_{i j} b_{i j}=1$, entonces:

$$
I=\Sigma_{i j} b_{i j} \log \frac{b_{i j}}{a_{i j}}
$$

- Se ha adoptado la convención de escribir $x$ para la matriz diagonal obtenida del vector $x$. Todos los vectores serán columnas; los acentos denotarán transposición. 
es minimizada bajo $\Sigma_{i} b_{i j}=v_{j}, \Sigma_{j} b_{i j}^{\prime}=u_{i}$, digamos, cuando $B$ es una transformada RAS de $A,(2.1)$ puede interpretarse como la información obtenida de una distribución bivariada a posteriori $\left(b_{i j}\right)$ dada una distribución a priori $\left(a_{i j}\right)$; este enfoqua fue propuesto por el presente autor, por de Leeuw y Theil [1966].

Si el logaritmo en (2.1) se expande, el término dominante es una ji-cuadrada; por lo tanto el RAS es aproximadamente un minimizador de ji-cuadrada; el criterio de la ji-cuadrada ha sido propuesto para ajustar matrices de frecuencias a sumas marginales conocidas por Deming y Stephan [1940] y Friedlander [1961].

Ninguna de estas interpretaciones "minimizadoras" tiene carácter económico. El profesor Stone llama a $r_{i}$ un "efecto sustitución" y a $s_{j}$ un "efecto de fabricación". También es posible ver el RAS a la luz de modelos gravitacionales de W. Isard. Se propone aquí otra interpretación, estrictamente económica, del RAS.

Si se consideran los coeficientes de insumo-producto como consecuencia de la maximización de beneficios, dada una función producción y un conjunto de precios exógenos de insumos y factores (como se deriva de los teoremas de no-sustitución tales como el de Klein [1952] en el concexto de la Cobb-Douglas, usado de hecho, por ejemplo por Morishima y Murata [1972] y Saito [1972]), los coeficientes físicos están dados por:

$$
a_{i j}=p_{j} \alpha_{i j} / p_{i}
$$

en la Cobb-Douglas, y por:

$$
a_{i j}=\left(p_{j} \theta_{i j} / p_{i}\right)^{\sigma j}
$$

en la CES, donde $\alpha_{i j}$ es el exponente para el insumo (o factor) $i$ en la función de producción del sector $j$ y $\theta_{i j}$ son los parámetros de distribución y $\sigma_{j}$ la elasticidad de sustitución en la función producción CES del sector $j$; los $p_{i}$ son los precios.

Extensiones directas del teorema de no-sustitución de Klein muestran que las funciones de producción de dos etapas, tales como la CES generalizada de Uzawa [1962] pueden tratarse de manera semejante. Considérese primero la mezcla CES de componentes Cobb-Douglas:

$$
X_{j}=\left\{\Sigma_{g}\left(\theta_{g j} \prod_{i_{\varepsilon}{ }^{S g}} X_{i j}{ }^{-\alpha_{i j} \rho_{g j}}\right)\right\}^{-1 / \rho_{g j}}
$$

donde $S_{g}$ es el conjunto de subíndices $i$ tales que el g-ésimo insumo de la "etapa" CES está formado por insumos numerados $i$, los $i \varepsilon S g$ de la "etapa" Cobb-Douglas. La maximización de ganancias lleva a:

$$
a_{i j}=\frac{p_{j} \alpha_{i j}}{p_{j}} \theta_{g j} A_{g j}-\left(\frac{1^{-} \sigma_{g j}}{\sigma_{g j}}\right)
$$

Nótese que el primer componente multiplicativo $p_{j} \alpha_{i j} / p_{j}$ es un coeficiente de insumo Cobb-Douglas. Si $A_{g j}$ es consistente con la maximización de 
utilidades en la CES: $A_{g j}=\left(\theta_{g j} p_{j} / P_{g}\right)_{g i}^{\sigma}$ y por lo tanto:

$$
a_{i j}=\left(P_{g} \alpha_{i j} / p_{i}\right)\left(p_{j} \theta_{g j} / P_{g}\right)^{\sigma_{g j}}
$$

el producto de un coeficiente Cobb-Douglas dentro de $S_{g}$ y los coeficientes CES entre los $S_{g}$. $P_{g}$ es el precio agregado $P_{g}=\Sigma_{i \varepsilon s g}\left(x_{i j} / x_{g j}\right) p_{i}, x_{g j}$ el g-ésimo componente Cobb-Douglas de la CES (2.4).

De manera semejante, una mezcla Cobb-Douglas de componentes CES:

$$
x_{j}=\prod_{g}\left\{\Sigma_{i} \varepsilon_{S_{g}}\left(\theta_{i j} x_{i j}{ }^{-\rho} g j\right)^{-1 / \rho_{g i}}\right\}_{g j}^{\alpha_{g j}}
$$

conduce de manera directa a:

$$
a_{i j}=\left(\theta_{i j} P_{g} / p_{i}\right)_{g j}^{\sigma}\left(p_{j} \alpha_{g j} / P_{g}\right)
$$

De acuerdo con (2.2), variaciones en el precio sin variación en la función de producción llevan a:

$a_{i j}(t+1)=\left\{p_{i}(t) / p_{i}(t+1)\right\} a_{i j}(t)\left\{p_{j}(t+1) / p_{j}(t)\right\}$

esto es, $r_{i}^{C D}=p_{i}(t) / p_{i}(t+1), s_{j}^{C D}=p_{j}(t+1) / p_{j}(t)$. A estos valores, $r_{i}^{C D}, s_{j}^{C D}$ les llamo coeficientes RAS-Cobb-Douglas. Directamente puede verse que (2.3) implica coeficientes RAS-CES:

$$
\begin{aligned}
& r_{i}^{C E S}=\left(r_{i}^{C D}\right)^{\sigma}{ }_{j} \\
& s_{j}^{C E S}=\left(s_{j}^{C D}\right)^{\sigma}{ }_{j}
\end{aligned}
$$

Desde luego, $r_{i}$ es independiente de $j$ ya sea en el caso de la CobbDouglas o entre sectores con la misma elasticidad de sustitución.

Los coeficientes definidos en (2.5) varian de acuerdo con:

$$
a_{i j}(t+1)=r_{i}^{C E S} \mathbf{r}_{\mathrm{g}}^{C D} a_{i j}(t) s_{g}^{C E S} s_{j}^{C D}
$$

y los de (2.6) de acuerdo con

$$
a_{i j}(t+1)=r_{i}^{C E S} r_{g}^{C D} a_{\mathrm{ij}}(t) s_{g}^{C E S} s_{j}^{C D}
$$

Algunos resultados empíricos sobre esto serán vistos en la sección VII.

III. El PROCEDimiento de ESTimación DE LAS MATRICES DE INSUMOPRODUCTO

La única matriz de insumo-producto completa para México fue recopilada en 1960 por el Banco de México. ${ }^{3}$ Esta será referida como la

\footnotetext{
"Banco de México, "Cuadro de insumo-producto para 1960", sin fecha.
} 
"matriz original de 1960 ". Otras fuentes de información son las cuentas nacionales, ${ }^{4}$ un cuadro no publicado sobre composición de importaciones, ${ }^{5}$ referida como "Importación" y el estudio conjunto de CEPAL y Nacional Financiera, ${ }^{6}$ referido como CEPAL-NAFINSA.

El modelo mexicano de insumo-producto incluye 46 sectores intermedios detallados en el cuadro 4, sección VI, y cuatro sectores de insumos primarios (importaciones, trabajo, capital e impuestos indirectos menos subsidios).

La demanda final se divide en consumo, exportaciones y formación bruta de capital (más o menos variaciones en inventarios). Las cuentas nacionales dan 50 renglones de totales a precios corrientes y a precios constantes de 1960 hasta 1967, salvo el renglón 47 (importaciones), el que se ha deflacionado utilizando índices de CEPAL-NAFINSA. Los totales de los renglones 1 a 46 para 1968/1972 a precios corrientes y constantes de 1960 también están dados en las cuentas nacionales. El total del renglón 47 está deflacionado mediante cifras inéditas de la CEPAL. Los totales de los renglones 48 a 50 están estimados de la manera siguiente: los impuestos indirectos son una proporción constante del $P I B(4.84 \%)$. El ingreso disponible está distribuído entre trabajo y capital:

$$
\begin{aligned}
& W_{t}=-.011356+.62303 w_{t-1}+.389621 w_{t-2}+.00638 w_{t-3} \\
& \begin{array}{llll}
(.167) & (.931) & (1.030) & (.125)
\end{array}
\end{aligned}
$$

$\left(R^{2}=0.999364\right)$ donde $w_{t}$ es la participación del trabajo en el año $t$.

Las sumas de las columnas hasta el renglón $47\left(\Sigma_{i=1}^{47} a_{i j}\right)$, de la columna 1 a la 46 , están dadas por las cuentas nacionales, a precios corrientes y de 1960; las exportaciones (el total de la columna 48) están dadas sólo a precios corrientes y deflacionadas con números índices de CEPAL-NAFINSA hasta 1969 y cifras no publicadas de CEPAL en 19701972. El total de la columna 49 (formación de capital bruto) está dado a precios corrientes y deflacionado con el deflator implícito del $P I B$; el consumo está estimado como un residuo del PIB. (+ importaciones-exportaciones-formación del capital bruto). Todo ésto cubre el período $1950-1972$.

Los estimadores de las matrices son RAS-equivalentes en dos etapas a la matriz original de 1960 en el sentido descrito después. Para el propósito de las estimaciones vía RAS, las matrices intermedias incluyen exportaciones, llamamos a ésta la matriz intermedia aumentada (MIA); la matriz de insumos primarios reducida (MPR) excluye las importaciones. Entonces, estando todas las matrices a precios constantes de 1960:

\footnotetext{
- "Cuentas nacionales y acervos de capital", sin fecha. Estadísticas de la Oficina de Cuentas de Producción y Precios, 1973. Incluye un Apéndice Estadístico publicado en octubre de 1973 .

"Importación de mercancías". Oficina de Cuentas del Exterior, octubre de 1973.

- CEPAL y Nacional Financiera, La política industrial en el desarrollo económico de México. NAFINSA, 1971.
} 
1) Para $t=1960$ la MIA de etapa I es RAS-equivalente a la matriz original de 1960 , bajo los totales de los renglones y columnas de las cuentas nacionales.

2) Para $t=1961$ a $t=1972$, la MIA de la etapa I para $t$ es RAS-equivalente a la MIA de etapa I para $t-1$, bajo los totales de los renglones y columnas de las cuentas nacionales.

3) Para $t=1950$ a $t=1959$, la MIA de la etapa I es RAS-equivalente a la MIA de etapa I para $t+1$, bajo los totales de renglones y columnas de las cuentas nacionales.

4) La MPR de la etapa I está dada en las cuentas nacionales para 1950-1967.

5) La MPR de la etapa I para 1968-1972 es RAS-equivalente para cada $t$ a la MPR de etapa I para $t-1$, bajo (3.1) y la distribución resultante del $P I B$.

6) El renglón 47 de la MIA de etapa II se define como sigue: para sectores intermedios, el elemento $(47, j)$ es el total del renglón 47 por la participación de los bienes intermedios en el total de importaciones (dada por "Importación") por la participación del sector $j$ en el renglón 47 de la etapa I. Esto cubre de $j=1$ a 46. Las importaciones de bienes de consumo $(47,47)$ y bienes de capital $(47,49)$ están definidas de manera semejante.

7) La columna 48 de etapa II incorpora la información de CEPALNAFINSA sobre las exportaciones de 16 grupos de bienes.

8) La submatriz resultante en la MIA de etapa II es RAS-equivalente a la sub-matriz correspondiente de la MIA de etapa I, bajo las cuentas nacionales (menos el valor de los elementos en el renglón 47 o columna 48 definidos antes).

9) Esto cubre de 1950 a 1969 . La MIA etapa II para $t$ es RAS-equivalente a la MIA de etapa II para $t-1$, con $t$ de 1970 a 1972.

10) Las matrices de 1973 a 1975 son todas RAS-equivalente a la matriz de $(t-1)$ con coeficientes del RAS definidos como sus valores medios de la etapa I para 1960-1969.

\section{UNA PRUEBA DE LA CALIDAD DE LAS PREDICCIONES DEL RAS}

Sólo pueden hacerse pruebas muy restringidas sobre la calidad de una matriz ajustada vía RAS; la única conclusiva implica tener una matriz "real" para el período que se predice. Se puede probar la extrapolación de una matriz a períodos en que los marginales no se conocen; sean $\widehat{r}_{j}$ y $\hat{s}_{j}$ los últimos valores conocidos de $r_{i}$ y $s_{j}$, digamos aquéllos que llevan a $A(t)$ en $A(t+1)$. Se puede tratar $A(t+2)=\hat{\bar{r}}^{2} A(t) \hat{\bar{s}}^{2}$, o en general, $A(t+h)=\hat{\vec{r}}^{h} A(t) \hat{s}^{h}$.

Se llevó a cabo una prueba sencilla siguiendo estos lineamientos, ya que las matrices de 1973, 1974 y 1975 están definidas del modo antes mencionado, al utilizar la matriz de 1972 y los valores promedio de $r$ y 
$s$ para 1960-1969. Las matrices intermedias de transacciones de 1970, 1971 y 1972 se predijeron usando la matriz de 1969 y las medias de $r$ y $s$ para 1960-1969; los totales marginales se compararon con los valores reales de las cuentas nacionales.

Parece ser que, por una parte, los totales de los renglones y columnas fueron predichos con más o menos el mismo grado de éxito, aunque los errores de las columnas son un poco mayores (véase el cuadro 1).

La raíz del error cuadrático medio (RECM):

$$
\left\{\frac{1}{46} \sum_{i=1}^{46}\left(\frac{x_{i}^{\rho}-x_{i}^{A}}{x_{i}^{4}}\right)^{2}\right\}
$$

donde $x_{i}^{\rho}$ es el valor predicho y $x_{i}^{A}$, el valor real de $x_{i}$, se calculó para los totales de renglón y columna en los tres años. Los errores crecen de manera considerable con el tiempo: la producción bruta, por ejemplo, se predijo en promedio con un error (RECM) de $8.35 \%$ a un año de distancia, $15.80 \%$ a dos años y $23.5 \%$ a tres años.

Cuadro 1

RAÍZ DEL ERROR CUADRÁTICO MEDIO PARA LOS

- MARGINALES PREDICHOS DEL RAS

(porcientos)

\begin{tabular}{lrrr}
\hline & 1970 & 1971 & 1972 \\
\hline Producoibn bruta total & & & \\
Sin oinoo dectores & 5.3538 & 15.8044 & 23.4878 \\
& 11.705 & 10.2817 & 12.1362 \\
Insumos intermedios totales & 11454 & 17.9810 & 26.3472 \\
Sin oinoo geotores & 9.7860 & 12.7193 & 15.2139 \\
\hline
\end{tabular}

Por otra parte, cinco sectores parecen dar cuenta de una gran parte de los errores de predicción; en los cinco casos los errores son de sobre-estimación. En el cuadro 2 se muestran los valores de $\left(x_{i}^{p}-x_{i}^{A}\right) / x_{i}^{A}$ para estos cinco sectores.

El período 1970-1972 es difícil para la economía mexicana. En 1970 se experimentó un cambio de administración, acontecimiento que, tradicionalmente, se piensa tiene consecuencias significativas. El año de 1971 fue de semi-recesión, cuando la tasa de crecimiento del $P I B$ bajó a la mitad; por su parte 1972 se considera como el año inicial de un período inflacionario moderado - una experiencia no conocida por largo tiempo en la economía mexicana. Se puede ver que los sectores llamados "tradicionales" continúan creciendo y que fueron subestimados por el RAS; 
los sectores "dinámicos" fueron en general sobre-estimados. Este es un tema interesante para una investigación ulterior.

Cuadro 2

ERroRES DE PREDICCIÓN PARA ALGUNOS SECTORES CRUCIALES

(porcientos)

\begin{tabular}{|c|c|c|c|c|c|c|}
\hline \multirow{2}{*}{$\begin{array}{l}\text { Perfodo } \\
\text { Seotor }\end{array}$} & \multicolumn{3}{|c|}{ Produoolon bruta } & \multicolumn{3}{|c|}{ Ingurios intermedion } \\
\hline & 1970 & 1971 & 1972 & 1970 & 1971 & 3972 \\
\hline \multirow{4}{*}{$\begin{array}{l}\text { Minerales no } \\
\text { netálicos } \\
\text { Qufmios báeioa } \\
\text { Abonos y } \\
\text { fertilizantea } \\
\text { otros productos } \\
\text { qufmioos } \\
\text { Mequinaria } \\
\text { eléotrios }\end{array}$} & $\begin{array}{l}19.1964 \\
16.4565\end{array}$ & $\begin{array}{l}32.2034 \\
36.4362\end{array}$ & $\begin{array}{l}67.8571 \\
69.9495\end{array}$ & $\begin{array}{l}16.0494 \\
22.4771\end{array}$ & $\begin{array}{l}25.8824 \\
47.1522\end{array}$ & $\begin{array}{l}54.3210 \\
88.7550\end{array}$ \\
\hline & 31.6384 & 51.0000 & 78.8546 & 32.8244 & 54.7973 & 86.3095 \\
\hline & 17.8451 & 24.8408 & 32.1637 & 20.5882 & 28.7037 & 39.5652 \\
\hline & 12.3306 & 39.6601 & 50.8750 & 12.4668 & 38.9503 & 50.9804 \\
\hline
\end{tabular}

Dejando pendiente una extensión de la prueba examinada (existen 20 años para hacer lo mismo), se podría concluir que la extrapolación del RAS a corto plazo, tal vez auxiliada con alguna hipótesis exógena (tal como la tasa de crecimiento del $P I B$, importaciones, exportaciones, etc.) puede no ser tan mala, si es que se mantiene contacto con algunos sectores cruciales, que parecen dar cuenta de una gran parte de los errores de predicción.

\section{Predicción de la demanda intermedia}

En esta sección nos ocupamos del poder predictivo del predictor de la demanda intermedia:

$$
z_{t h}^{P}=\left[\left(I-A_{t}\right)^{-1}-I\right] \delta_{t+h}
$$

Tilanus [1966], Tilanus y Rey [1963], Rey y Tilanus [1964] y Theil [1966] han estudiado este campo extensamente para la economía holandesa; también pueden estudiarse insumos primarios; véase Tịlanus y Harkema [1962].

Desde luego, nuestro experimento es bastante artificial; todas las matrices $A_{t}$ son estimadas y el proceso de estimación incluye $\delta_{t}$. Vale la pena señalar que es improbable que los errores de (5.1) estén sobreestimados, al menos en un sentido medio, ya que las estimaciones de las matrices minimizan ${ }^{7}$ la variación media de éstas, en el sentido de la teoría de la información.

Los estudios holandeses entran en muchos detalles que dejaremos

${ }^{7}$ No del todo, ya que el proceso de estimación no es directamente el RAs, pero es semejante. 
de lado; no se busca aquí la estructura estadística de los errores de predicción, sino una medición de la eficiencia de una matriz de coeficientes "vieja" para predecir la demanda intermedia, dado un pronóstico perfecto de la demanda final.

Interesa comparar la actuación de (5.1) contra un predictor más elemental, que también parte de una predicción perfecta de demanda final, pero no supone el conocimiento de los coeficientes de insumos:

$$
z_{i t h}^{B}=z_{i t}\left(\frac{\delta_{i}, t+h}{\delta_{i t}}\right)
$$

El predictor (5.2) ha sido llamado predictor "de expansión de la demanda final". Llamaremos a (5.1) el predictor de "insumo-producto".

Estamos interesados en el comportamiento de las raíces de los errores cuadráticos medios:

$$
\begin{aligned}
& e_{i h}^{P}=\frac{1}{T-h}\left[\sum_{t=1}^{T-h}\left(\frac{z_{i t h}^{P}-z_{i}, t+h}{z_{i, t+h}}\right)^{2}\right]^{1 / 2} \\
& e_{i h}^{B}=\frac{1}{T-h}\left[\sum_{i=1}^{T-h}\left(\frac{z_{i t h}^{B}-z_{i}, t+h}{z_{i, t+h}}\right)^{2}\right]^{1 / 2}
\end{aligned}
$$

donde $T$ es la duración del período en análisis (aquí $T=26$ ).

La actuación del predictor de insumo-producto empeora con la duración del período de predicción, como era de esperarse, y existe una gran variación intersectorial en el comportamiento de $e_{i j}^{\rho}$. Por ejemplo, los sectores mineros alcanzan errores mayores del $10 \%$ en uno y dos años; "otros textiles" (fibras duras) y construcción en dos; fertilizantes en tres; química básica en seis; materiales sintéticos en cinco; silvicultura en seis; mientras que otros como la fabricación de alimentos, comunicaciones, comercio, caucho, imprenta y editorial o petróleo y petroquímica no alcanzan este nivel en todo el período dei análisis ( 25 años de horizonte). Los períodos críticos (en el sentido de errores de predicción menores del 10\%) son mayores para los servicios, salvo transportes.

Esta no es la pauta de conducta del predictor "de expansión": después de un punto crítico, seis años en promedio, los errores empiezan a declinar hasta un promedio de $18 \%$ para los 20 años y se elevan otra vez después (véase el cuadro3) ${ }^{8}$ Por lo pronto, la actuación a corto plazo del predictor de insumo-producto parece mejor que la del de expansión; la situación es contraria en el largo plazo (después de cua-

${ }^{8}$ Este no es el comportamiento encontrado en los estudios holandeses; véase Theil [1966]. p. 166. 
tro años en promedio): El cuadro 3 muestra los valores de:

$$
\begin{gathered}
\bar{e}_{h}^{P}=\frac{1}{46} \sum_{i=1}^{46} e_{i h}^{P} \\
\bar{e}_{h}^{B}=\frac{1}{46} \sum_{i=1}^{46} e_{i h}^{B}
\end{gathered}
$$

Los valores están fuertemente dominados por los errores de los sectores 5, 6 y 14, de tal manera que, a un lado el cuadro 3 presenta:

$$
\begin{aligned}
& \bar{e}_{h}^{, P}=\frac{1}{43}\left(i \neq \Sigma_{5,6,14} e_{i h}^{P}\right) \\
& \bar{e}_{h}^{, B}=\frac{1}{43}\left(i \neq \Sigma_{5,6,14} e_{h i}^{B}\right)
\end{aligned}
$$

Si se utilizan $\vec{e}_{h}^{, P}$ y $\bar{e}_{h}^{, B}$, el predictor de expansión actúa mejor que el de insumo-producto después de ocho años. La media sectorial de nivel crítico de $10 \%$ de error medio es de cuatro años para el predictor de insumo-producto y dos años para el de expansión si todos los sectores se toman en consideración; 11 años para el de insumo-producto y 12 para el de expansión si los sectores dominantes son excluidos.

\section{Cuadro 3}

\begin{tabular}{|c|c|c|c|c|}
\hline \multirow{2}{*}{$\begin{array}{c}\text { Años } \\
\text { adelante }\end{array}$} & \multicolumn{2}{|c|}{ Insumo - producto } & \multicolumn{2}{|c|}{$\begin{array}{l}\text { Expantión de la } \\
\text { demanda final }\end{array}$} \\
\hline & $A$ & $B^{a /}$ & A & $\mathrm{B}^{\mathrm{a} /}$ \\
\hline $\begin{array}{r}1 \\
2 \\
3 \\
4 \\
5 \\
6 \\
7 \\
8 \\
9 \\
10 \\
11 \\
12 \\
13 \\
14 \\
15 \\
16 \\
17 \\
18 \\
19 \\
20 \\
21 \\
22 \\
23 \\
24 \\
25\end{array}$ & $\begin{array}{l}0.020369 \\
0.041807 \\
0.076551 \\
0.415902 \\
0.154525 \\
0.191774 \\
0.197753 \\
0.204174 \\
0.237075 \\
0.262386 \\
0.298328 \\
0.325883 \\
0.371613 \\
0.449625 \\
0.510355 \\
0.481798 \\
0.468006 \\
0.465440 \\
0.527681 \\
0.639423 \\
0.805617 \\
1.04452 \\
1.317865 \\
1.259723 \\
1.295315\end{array}$ & $\begin{array}{l}0.016423 \\
0.027270 \\
0.042152 \\
0.056720 \\
0.061742 \\
0.062417 \\
0.070831 \\
0.079390 \\
0.087272 \\
0.097593 \\
0.109508 \\
0.121670 \\
0.134517 \\
0.146087 \\
0.158047 \\
0.172252 \\
0.182266 \\
0.198109 \\
0.207107 \\
0.223229 \\
0.247337 \\
0.277170 \\
0.326539 \\
0.393608 \\
0.413919\end{array}$ & $\begin{array}{l}0.076105 \\
0.257118 \\
0.488365 \\
0.694574 \\
0.898923 \\
1.015625 \\
0.624096 \\
0.319685 \\
0.308324 \\
0.258630 \\
0.233216 \\
0.238642 \\
0.283794 \\
0.311779 \\
0.307058 \\
0.218269 \\
0.188489 \\
0.157746 \\
0.146353 \\
0.182646 \\
0.235497 \\
0.290216 \\
0.366483 \\
0.384313 \\
0.209831\end{array}$ & $\begin{array}{l}0.018927 \\
0.031225 \\
0.053787 \\
0.075978 \\
0.096592 \\
0.081417 \\
0.071478 \\
0.072247 \\
0.072575 \\
0.069537 \\
0.082903 \\
0.101889 \\
0.117014 \\
0.096908 \\
0.094017 \\
0.100588 \\
0.092035 \\
0.081595 \\
0.066511 \\
0.110780 \\
0.153827 \\
0.189102 \\
0.240061 \\
0.297411 \\
0.155952\end{array}$ \\
\hline
\end{tabular}

RAÍz DE LOS ERRORES CUADRÁTICOS MEDIOS DE PREDICCIÓN PARA PREDICTORES DE DEMANDA INTERMEDIA, DE INSUMO-PRODUCTO Y DE EXPORTACIÓN DE LA DEMANDA FINAL. POR DURACIÓN DEL HORIZONTE

axcluye los sectores 5, 6 y 14 


\section{Fuentes de la VARIACIÓN DE LA DEMANDA PARA INSUMOS} INTERMEDIOS: VARIACIÓN DE COEFICIENTES

Considérese la ecuación familiar de insumo-producto para demanda intermedia:

$$
z=x-\delta=\left\{(I-A)^{-1}-I\right\} \delta=A(I-A)^{-1} \delta=C \delta
$$

Al fechar $z, \delta$ y $A$ con el índice $t$, desfasando un período y restando se obtiene:

$$
\begin{aligned}
& z_{t}-z_{t-1}=C_{t} \delta_{t}-C_{t-1} \delta_{t-1}=C_{t}\left(\delta_{t}-\delta_{t-1}\right)+\left(C_{t}-C_{t-1}\right) \delta_{t-1} \\
& =C_{t-1}\left(\delta_{t}-\delta_{t-1}\right)+\left(C_{t}-C_{t-1}\right) \delta_{t}
\end{aligned}
$$

En lo que sigue se tomará la media simple de los dos miembros de la derecha de (6.2) y se definirá $C^{*}=\left(C_{t}+C_{t-1}\right) / 2, \delta^{*}=\left(\delta_{t}+\delta_{t-1}\right) / 2$, $\Delta C=C_{t}-C_{t-1}, \Delta \delta=\delta_{t}-\delta_{t-1}$, de tal manera que:

$$
\Delta z=z_{t}-z_{t-1}=C^{*} \Delta \delta+(\Delta C) \delta^{*}
$$

El primer término a la derecha es el efecto de los cambios en la demanda final, al mantener constante $C$ al valor $C^{*}$, sobre el cambio en la demanda intermedia. El segundo término es el efecto del cambio de coeficientes, con la demanda final constante en $\delta^{*}$. Cabe una palabra de precaución: éste no es un cambio tecnológico, como lo propuso H. Simon [1951]. Los coeficientes de insumo-producto son el resultado de dos cosas: una tecnología ex-ante (por ejemplo, como lo expresa la función producción neoclásica) y precios relativos de insumos y factores. Este tema será explorado en las secciones VII y VIII.

En el cuadro 4 se muestra el valor medio de los componentes de (6.3) relativos al cambio en la demanda intermedia: $(\Delta C) \delta^{*} / \Delta z$, y el signo de $C^{*} \Delta \delta / \Delta z$, expresados en porcientos, para los 10 períodos 1963 1964 a 1972-1973, para los 46 sectores intermedios.

Se muestran los signos de $C^{*} \Delta \delta / \Delta z$, aunque no son necesarios ya que en todos los casos con $(\Delta C) \delta^{*} / \Delta z$ positivo, su valor porcentual está por abajo de 100. Si se observa el número de signos negativos de $(\Delta C) \delta^{*}$ se encuentra que casi todos los sectores tradicionales tienen un gran número de ellos: agricultura y silvicultura tienen 9; "otros textiles" (fibras duras), cuero y productos del cuero, madera y corcho, alquiler de inmuebles y sorprendentemente, construcción tienen 8. Otra sorpresa son los 7 signos negativos en el sector petróleo.

Por otro lado, los sectores "modernos" (entre los cuales debería estar el petróleo) muestra un poco o ningún trazo de $(\Delta C) \delta^{*}$ negativos. En general, dejando a un lado cifras muy grandes (debido a $\Delta z$ muy pequeño) el cuadro 4 muestra que el cambio de coeficientes es un determinante no trivial de los cambios en la demanda intermedia.

Otra forma de ver esto es la siguiente: manténgase la matriz de coeficientes constante en $t=t_{0}$, y obsérvense únicamente los efectos de los 
Cuadro 4

COMPOSICÓN DE LOS CAMBIOS EN LA DEMANDA POR INSUMOS INTERMEDIOS (porcientos con respecto $a \Delta z, 1963-1964$ )

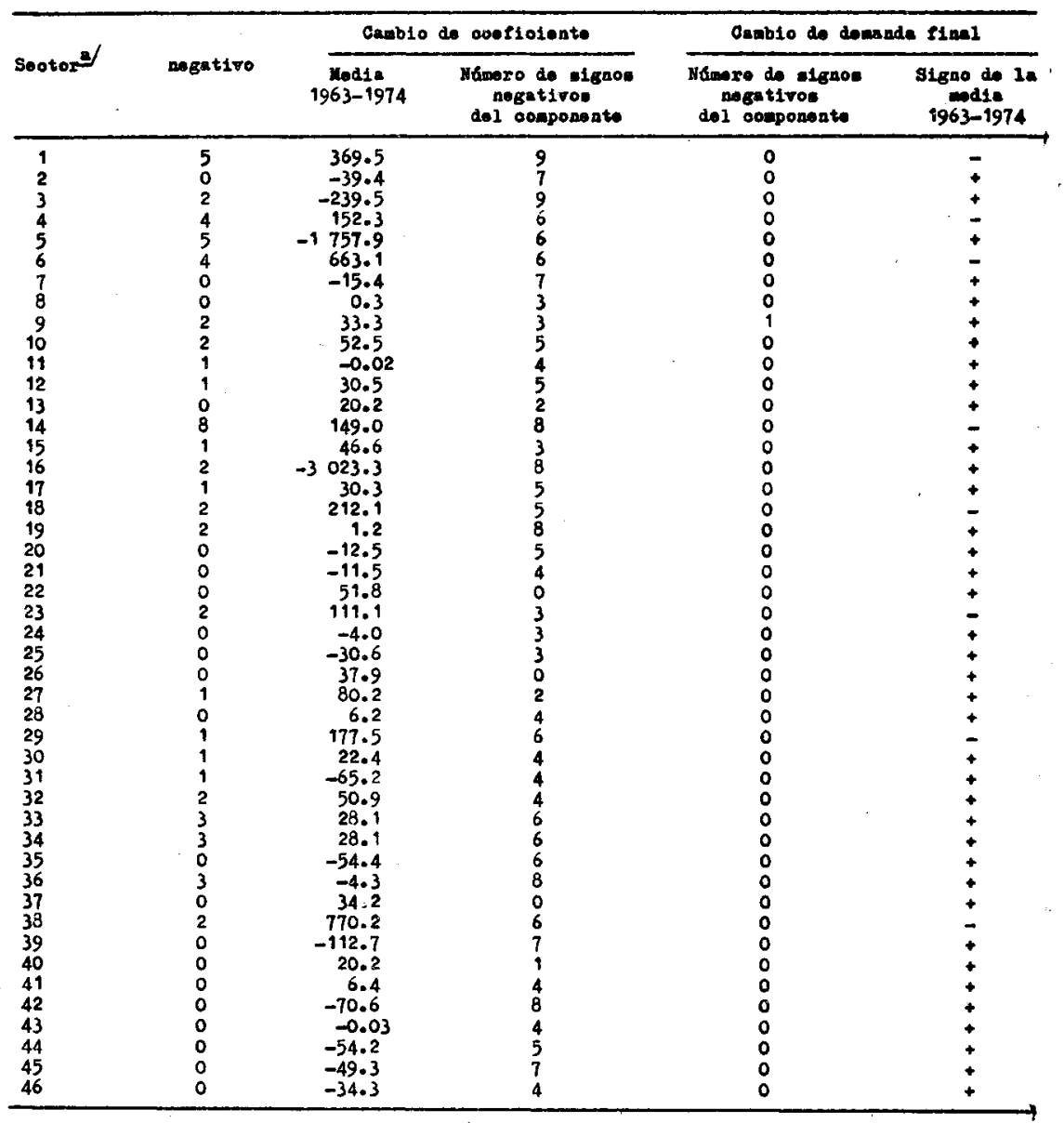

a Véase después del cuadro 6 la lista de sectores correspondientes 
cambios en la demanda final hasta $t=T$. El cuadro 5 muestra el resultado de hacer esto para $t_{0}=1963$ y $T=1973$; esto es, manteniendo la matriz para 1963 constante y buscando el valor de $z$ (o $x$ ) en 1973, dejando variar la demanda final, desde 1964 tal como se observó en la realidad. El cuadro 5 muestra la producción bruta observada de 1973, y el valor de la producción bruta en 1973 si es que la matriz de coeficientes intermedios se mantuviera constante en su valor de 1963. La relación entre los valores reales e hipotéticos de 1973 es la ganancia proporcional (o pérdida si es menor que 1) en la producción bruta de cada sector, debido a los cambios de coeficientes durante el decenio. Así, se encuentra pérdida en producción bruta de la agricultura a una tasa anual de $1.7 \%(1-.831195) / 10$; de la silvicultura a $2.25 \%$ al año; minería de metálicos al $4.2 \%$; minería de no metálicos al 3.13 . Otros textiles (fibras duras) al 4.3 ; mientras que el sector de química básica tiene ganancias de casi $1 \%$ al año; fibras sintéticas al 7.6; abonos y fertilizantes al 2.6; etc. Esto es bastante consistente con lo que puede observarse en el cuadro 4 . Se ve que los sorprendentes 7 signos negativos del sector petróleo, tienen pocas consecuencias: una tasa de pérdida de la producción bruta menor al uno porciento; se concluye que su considerable crecimiento se debe a la demanda final.

Se puede concluir que, como efecto de los precios o como efecto del cambio tecnológico, existe una clara sustitución de insumos "tradicionales" (productos agrícolas, minerales) por insumos sintéticos. En la siguiente sección se hará una investigación de las causas (precios y tecnología).

\section{Cambio tecnológico en un "Mundo" Cobb-Douglas}

En esta sección seguiremos a Klein [1952] y supondremos una función producción Cobb-Douglas que genera tanto coeficientes de insumo como de factores. Desde luego, todo esto es reducible a una función producción sólo con factores como argumentos, pero se ha visto que existen cambios importantes en los coeficientes intermedios y se tratará de obtener una explicación. Sólo se hablará aquí de los coeficientes de insumos.

Al utilizar los resultados de la sección II, se tiene la ecuación básica:

$$
a_{i j}(t+1)=r_{i}^{C D} a_{i j}(t) s_{j}^{C D}
$$

donde $r_{i}^{C D}=p_{i}(t) / p_{i}(t+1)$ y $s_{j}^{C D}=p_{j}(t+1) / p_{j}(t) ;$ llamamos a (7.1) el modelo Ras-Cobb-Douglas.

Nuestro propósito en esta sección es compararlo con el modelo RAS "ordinario", o sea $a_{i j}(t+1)$ obtenido del algoritmo de Stone, sujeto a los totales marginales de las cuentas nacionales. Otra vez se requerirá una manera de sintetizar una gran cantidad de información $(46 \times 46$ 
Cuadro 5

Producción BRUTA, 1973. Matriz de insumo Producto, REAL Y CONSTANTE A 1963

(millones de pesos de 1960)

\begin{tabular}{|c|c|c|c|c|}
\hline \multirow{3}{*}{ Seotor ${ }^{2}$} & \multicolumn{3}{|c|}{ Producoisn bruta } & \multirow{3}{*}{$\begin{array}{c}\text { Rolaoibn } \\
(2 / 3)\end{array}$} \\
\hline & \multirow[b]{2}{*}{$1963(1)$} & \multirow{2}{*}{ Re21, 1973 (2) } & \multirow{2}{*}{$\begin{array}{c}1973(3) \\
\text { ooeficiontea do } \\
1963\end{array}$} & \\
\hline & & & & \\
\hline 1 & 21762 & 26304 & 31646. & 0.831195 \\
\hline 2 & 12805 & 22020 & 22312 & 0.986913 \\
\hline 3 & 1056 & 1388 & 1792 & 0.774554 \\
\hline 4 & 676 & 902 & 1089 & $\begin{array}{l}0.828283 \\
0.58132\end{array}$ \\
\hline 5 & 3088 & $\begin{array}{ll}3 & 354 \\
2 & 390\end{array}$ & $\begin{array}{r}5770 \\
3 \\
3\end{array} 480$ & $\begin{array}{l}0.581282 \\
0.686782\end{array}$ \\
\hline 7 & $\begin{aligned} 11544 \\
11568\end{aligned}$ & $\begin{array}{r}2 \\
24890 \\
24\end{array}$ & $\begin{array}{rl}3 & 300 \\
25 & 109\end{array}$ & $\begin{array}{l}0.0001722 \\
0.991398\end{array}$ \\
\hline 8 & 4077 & 7807 & 7728 & 1.010223 \\
\hline 9 & $\begin{array}{l}40 \quad 107 \\
10\end{array}$ & 15021 & 14657 & 1.037115 \\
\hline 10 & 94400 & 26942 & 27467 & 0.980886 \\
\hline 11 & 4908 & 9649 & 9620 & 1.003015 \\
\hline 12 & 1405 & 2301 & 2302 & 0.999566 \\
\hline 13 & 5350 & 15592 & 14634 & 1.065464 \\
\hline $\begin{array}{l}14 \\
15\end{array}$ & $\begin{array}{l}1556 \\
5982\end{array}$ & $\begin{array}{rl}1 & 3338 \\
16 & 202\end{array}$ & $\begin{array}{r}2334 \\
1642\end{array}$ & $\begin{array}{l}0.573265 \\
0.98604\end{array}$ \\
\hline 16 & $\begin{array}{l}3962 \\
1983\end{array}$ & $\begin{array}{r}16202 \\
2008\end{array}$ & $\begin{array}{rl}16 & 422 \\
2 & 611\end{array}$ & $\begin{array}{l}0.986004 \\
0.769054\end{array}$ \\
\hline 17 & 2935 & 6518 & 6421 & 1.015107 \\
\hline 18 & 1903 & 4020 & 3586 & 1.008530 \\
\hline 19 & 1253 & 2159 & 2743 & 0.787094 \\
\hline 20 & 1417 & 3308 & 3393 & 0.974948 \\
\hline 21 & 1480 & 4449 & 4054 & 1.097435 \\
\hline $\begin{array}{l}22 \\
23\end{array}$ & 959 & $\begin{array}{ll}6 & 665 \\
2 & 536 \\
\end{array}$ & $\begin{array}{l}3795 \\
2015\end{array}$ & $\begin{array}{l}1.756258 \\
1.220560\end{array}$ \\
\hline 24 & $\begin{array}{ll}1 & 035 \\
1 & 155\end{array}$ & $\begin{array}{l}2530 \\
2582\end{array}$ & $\begin{array}{l}2015 \\
2617\end{array}$ & $\begin{array}{l}1.235860 \\
1.024838\end{array}$ \\
\hline 25 & 2394 & 5666 & 5525 & 1.025593 \\
\hline 26 & 910 & 2943 & 2746 & 1.071743 \\
\hline 27 & 1539 & 3753 & 3614 & 9.038463 \\
\hline 28 & 2910 & 8419 & 8084 & 1.041350 \\
\hline 29 & $\begin{array}{ll}0 \\
0\end{array}$ & 15122 & 15620 & 0.968118 \\
\hline 30 & 3200 & 7604 & 7801 & 0.974799 \\
\hline $\begin{array}{l}31 \\
32\end{array}$ & $\begin{array}{l}1149 \\
3\end{array}$ & $\begin{array}{l}4975 \\
99075\end{array}$ & $\begin{array}{l}4243 \\
8.777\end{array}$ & 9.172524 \\
\hline 33 & 1301 & $\begin{array}{l}9075 \\
2800\end{array}$ & $\begin{array}{l}8777 \\
2949\end{array}$ & 1.033894 \\
\hline 34 & 3774 & 15598 & $\begin{array}{rl}2 & 949 \\
15 & 191\end{array}$ & $\begin{array}{l}0.531702 \\
1.026799\end{array}$ \\
\hline 35 & 1778 & 3881 & 3761 & 1.031907 \\
\hline 36 & 16921 & 38814 & 39846 & 0.9741102 \\
\hline 37 & 3005 & 9206 & 7735 & 1.197986 \\
\hline 38 & 2332 & 3682 & 3672 & 1.002942 \\
\hline 39 & 793 & 14660 & 14877 & 0.985380 \\
\hline $\begin{array}{l}40 \\
41\end{array}$ & 1091 & 3023 & 2511 & 1.204206 \\
\hline $\begin{array}{l}41 \\
42\end{array}$ & 63274 & $126 \quad 645$ & 123337 & 1.026823 \\
\hline $\begin{array}{l}42 \\
43\end{array}$ & $\begin{array}{r}44932 \\
5 \\
257\end{array}$ & 23.33 & 24617 & 0.968290 \\
\hline $\begin{array}{l}43 \\
44\end{array}$ & $\begin{array}{ll}5 & 251 \\
3 & 589\end{array}$ & $\begin{array}{r}11 \\
8120 \\
8\end{array}$ & $\begin{array}{r}11231 \\
7822\end{array}$ & $\begin{array}{l}.0101697 \\
1.033800\end{array}$ \\
\hline 45 & 9518 & 15989 & 15615 & 1.023945 \\
\hline 46 & 13235 & 28725 & 27796 & 1.033423 \\
\hline
\end{tabular}

a Véase después del cuadro 6 la lista de sectores correspondientes 
coeficientes por 25 años $), a_{i j}(t+1)$ los coeficientes obtenidos del RAS ordinario. Si el modelo de Cobb-Douglas se sostiene, la discrepancia con (7.1) se explica como un cambio en $\alpha_{i j}$; sabemos que $\alpha_{i j}$ es el coeficiente monetario $p_{i} a_{i j} / p_{j}$; por lo tanto se puede estimar

$$
\begin{aligned}
\frac{\alpha_{i j}(t+1)}{\alpha_{i j}(t)}= & \frac{p_{i}(t+1) \alpha_{i j}(t+1) p_{j}(t)}{p_{j}(t+1) \alpha_{i j}(t) p_{i}(t)} \\
& =r_{i} s_{j} / r_{i}^{C D} s_{j}^{O D}
\end{aligned}
$$

donde $r_{i}$ y $s_{j}$ son coeficientes RAS ordinarios.

El cuadro 6 muestra las medias anuales, de 1963 a 1972 de los valores medios de $a_{i j}(t+1) / a_{i j}(t)$ a lo largo de los renglones (coeficientes de producción) y coiumna (coeficientes de insumos). Muestra también los valores medios del cambio predicho bajo supuestos Cobb-Douglas. A partir de ahí se obtiene una aproximación a (7.2). Ciertamente, el cambio predicho por la Cobb-Douglas es $r_{i}^{C D} s_{j}^{C D}$, de tal manera que (7.2) es la relación de RAS ordinario al RAS Cobb-Douglas. Aproximo la media de (7.2) por el cociente de la media de las variaciones del RAS ordinario a la media de las variaciones del RAS Cobb-Douglas.

Los cambios en los renglones están relacionados con incrementos en la demanda intermedia del insumo $i$; la media de renglón de (7.2) mostrará la razón media de incremento anual (si es mayor que 1) en la utilización del insumo $i$, independiente de las variaciones de los precios. $\mathrm{El}$ cambio predicho Cobb-Douglas mostrará la parte del incremento en la utilización de $i$, debida a un precio relativo más bajo (si es $>1$ ); el resultado (multiplicativo) final es el cambio predicho por el RAS.

Los cambios a lo largo de la columna pueden denotar varias cosas: si el cambio es menor que 1, la media de (7.2) mostrará que la tecnología del sector $j$ (función producción ex-ante) consume menos insumos intermedios. Haciendo uso muy intensivo de la imaginación, se puede interpretar este componente como el complemento de una tasa de incremento anual de la productividad. No estoy dispuesto a aventurar una interpretación tan debatible. La predicción vía la Cobb-Douglas mostrará el incremento esperado (o decremento) en el uso de insumos físicos debido a cambios en los precios; si es menor que uno, mostrará que el precio del producto se ha elevado por arriba del precio de los insumos, y viceversa. El resultado final es el cambio predicho por el RAS que muestra el incremento o decremento real (que es el producto de cambios debidos a los precios y cambios debidos a la tecnología).

Del examen del cuadro 6 se concluye que, aún si casi todos los cambios son pequeños, existe una consistencia notable con los resultados de la sección VI. Se. encuentra que los cambios predichos por la Cobb-Douglas son muy pequeños y que los sectores "tradicionales" muestran una tasa considerable de cambio ex-ante (agricultura $6.5 \%$ al año, minería 
Cuadro 6

VARIACIONES DE LOS COEFICIENTES RAS ORDINARIOS Y COBB-DOUGLAS; VARIACIONES ex-ante, APROXIMADOS (media en el tiempo de variaciones proporcionales; medias de renglón y columna)

\begin{tabular}{|c|c|c|c|c|c|c|}
\hline \multirow{2}{*}{ Sactorl } & \multicolumn{3}{|c|}{ Coefiolontas de produooton } & \multicolumn{3}{|c|}{ Cooftelontes do inoumo } \\
\hline & Rus & $\begin{array}{l}\text { Cobb- } \\
\text { Douglas }\end{array}$ & $\begin{array}{l}\text { or-ante } \\
\text { (aproxinadau) }\end{array}$ & Rus & $\begin{array}{c}\text { Cobb- } \\
\text { Douglas }\end{array}$ & $\begin{array}{c}\text { ox-ante } \\
\text { (aproximadan) }\end{array}$ \\
\hline $\begin{array}{r}1 \\
2 \\
3 \\
4 \\
5 \\
6 \\
7 \\
8 \\
9 \\
10 \\
11 \\
12 \\
13 \\
14 \\
15 \\
16 \\
17 \\
18 \\
19 \\
20 \\
21 \\
22 \\
23 \\
24 \\
25 \\
26 \\
27 \\
28 \\
29 \\
30 \\
31 \\
32 \\
33 \\
34 \\
35 \\
36 \\
37 \\
38 \\
39 \\
40 \\
41 \\
42 \\
43 \\
44 \\
45 \\
46\end{array}$ & $\begin{array}{l}0.931723 \\
0.991286 \\
0.959341 \\
0.944377 \\
0.915238 \\
0.951225 \\
1.008422 \\
1.001062 \\
0.984064 \\
0.975993 \\
1.005922 \\
0.995057 \\
1.032729 \\
0.921024 \\
1.033424 \\
0.978263 \\
1.005950 \\
1.0003600 \\
0.975897 \\
1.008652 \\
1.014258 \\
1.101757 \\
1.029184 \\
1.026202 \\
1.018786 \\
1.055997 \\
1.008585 \\
1.018246 \\
1.003134 \\
1.008001 \\
1.066220 \\
1.033827 \\
0.994109 \\
1.058586 \\
1.029775 \\
0.973637 \\
1.045044 \\
1.006413 \\
1.007250 \\
1.035518 \\
1.013760 \\
0.988303 \\
1.021269 \\
1.009613 \\
0.992274 \\
1.0002181\end{array}$ & $\begin{array}{l}0.995898 \\
0.993453 \\
0.9992927 \\
1.027358 \\
1.032182 \\
1.008807 \\
0.979757 \\
1.004097 \\
0.985222 \\
1.006650 \\
1.016248 \\
0.9992558 \\
0.994724 \\
1.003371 \\
1.02873 \\
0.983850 \\
0.996640 \\
0.994312 \\
1.020077 \\
0.971425 \\
0.981641 \\
0.949761 \\
0.960659 \\
0.988907 \\
0.985221 \\
0.984600 \\
0.977082 \\
1.000810 \\
0.985746 \\
0.999303 \\
0.999318 \\
0.992305 \\
1.034779 \\
0.984474 \\
1.003403 \\
1.012945 \\
0.970323 \\
1.025257 \\
0.983410 \\
0.999801 \\
1.003511 \\
1.012572 \\
1.015265 \\
1.020183 \\
1.043743 \\
1.012957\end{array}$ & $\begin{array}{l}0.935561 \\
0.997819 \\
0.966174 \\
0.919229 \\
0.886789 \\
0.942921 \\
1.029257 \\
0.996977 \\
0.998824 \\
0.975359 \\
0.989839 \\
1.002518 \\
1.038207 \\
0.917930 \\
1.090315 \\
0.999321 \\
1.009341 \\
1.009341 \\
0.956690 \\
1.038322 \\
1.033227 \\
1.160036 \\
1.071331 \\
1.037713 \\
1.034068 \\
1.072422 \\
1.032242 \\
1.017422 \\
1.017639 \\
1.008704 \\
1.066948 \\
1.041844 \\
0.960692 \\
1.075280 \\
1.026283 \\
0.961194 \\
1.077006 \\
0.981620 \\
1.024242 \\
1.037241 \\
1.010214 \\
0.976032 \\
1.005913 \\
0.989639 \\
0.950688 \\
0.989362\end{array}$ & $\begin{array}{l}1.101504 \\
1.115847 \\
1.062003 \\
1.085964 \\
1.135180 \\
1.076140 \\
1.052706 \\
1.069711 \\
1.098506 \\
1.105121 \\
1.072753 \\
1.077406 \\
1.061889 \\
1.107976 \\
1.060727 \\
1.084358 \\
1.065985 \\
1.062540 \\
1.066214 \\
1.042108 \\
1.068200 \\
1.042744 \\
1.068010 \\
1.066132 \\
1.048184 \\
1.058008 \\
1.080512 \\
1.062930 \\
1.075779 \\
1.066057 \\
1.071935 \\
1.047592 \\
1.068490 \\
1.053750 \\
1.067475 \\
1.062212 \\
1.028329 \\
1.061248 \\
1.058311 \\
1.051876 \\
1.054345 \\
1.063765 \\
1.078929 \\
1.089968 \\
1.059866 \\
1.030892\end{array}$ & $\begin{array}{l}1.064676 \\
1.064936 \\
1.069831 \\
1.028348 \\
1.027369 \\
1.055968 \\
1.085403 \\
1.058522 \\
1.075298 \\
1.059708 \\
1.037651 \\
1.068084 \\
1.067799 \\
1.059589 \\
1.037537 \\
1.078522 \\
1.063188 \\
1.067623 \\
1.037287 \\
1.094192 \\
1.082579 \\
1.119669 \\
1.104836 \\
1.075100 \\
1.078394 \\
1.079487 \\
1.086084 \\
1.062106 \\
1.077620 \\
1.062848 \\
1.062396 \\
1.073063 \\
1.027840 \\
1.081183 \\
1.051852 \\
1.048222 \\
1.098521 \\
1.038058 \\
1.080776 \\
1.062189 \\
1.059972 \\
1.0499778 \\
1.047135 \\
1.040966 \\
1.018779 \\
1.051019\end{array}$ & $\begin{array}{l}1.034591 \\
1.047807 \\
0.992683 \\
1.056028 \\
1.104939 \\
1.019103 \\
0.969876 \\
1.010627 \\
1.021583 \\
1.042854 \\
1.033828 \\
1.008728 \\
0.994465 \\
1.045666 \\
1.022351 \\
1.005411 \\
1.002631 \\
0.995239 \\
1.027887 \\
0.952400 \\
0.986718 \\
0.931297 \\
0.961954 \\
0.991658 \\
0.971986 \\
0.990101 \\
0.994925 \\
1.000776 \\
0.998292 \\
1.003019 \\
1.008979 \\
0.0976263 \\
1.039549 \\
0.974677 \\
1.014853 \\
1.013346 \\
0.936103 \\
1.022340 \\
0.979214 \\
0.990291 \\
0.994691 \\
1.013324 \\
1.030363 \\
1.047074 \\
1.040330 \\
0.980850\end{array}$ \\
\hline
\end{tabular}

a Véase la lista anexa de sectores correspondientes. 
SECTORES DE LA MATRIZ DE INSUMO-PRODUCTO

1. Agricultura

2. Ganadería

3. Silvicultura

4. Pesca

5. Minerales metálicos

6. Minerales no metálicos

7. Petróleo y petroquímica

8. Matanza de ganado y lácteos

9. Molienda de trigo y maíz

10. Otros productos alimenticios

11. Bebidas

12. Manufactura del tabaco

13. Hilados y tejidos

14. Otros textiles (fibras duras)

15. Vestuario y calzado

16. Madera y corcho

17. Papel y producto de papel

18. Imprenta y editoriales

19. Cuero y productos del cuero

20. Productos de hule

21. Productos químicos básicos

22. Fibras sintéticas

23. Fertilizantes e insecticidas

24. Jabones y detergentes

25. Productos farmacéuticos

26. Cosméticos y perfumes

27. Otros productos químicos

28. Productos minerales no metálicos

29. Industria metalúrgica básica

30. Fabricación de productos metálicos

31. Maquinaria no eléctrica

32. Maquinaria eléctrica

33. Material de transporte

34. Industria automotriz

35. Manufacturas diversas

36. Construcción

37. Electricidad

38. Cinematografía

39. Transportación

40. Comunicación

41. Comercio

42. Inmuebles

43. Hoteles y restaurantes

44. Servicios bancarios

45. Otros servicios

46. Gobierno 
de metálicos 11.4, minería de no metálicos 5.8) que lleva a un decremento en los coeficientes medios de producción. Se predice decrecimiento del uso de insumos petroleros, si se parte de los precios (pronóstico Cobb-Douglas), pero la cifra observada crece debido a cambios ex-ante. Los sectores "modernos" muestran la misma pauta que la de la sección VI: los materiales sintéticos son absorbidos a una tasa media ex-ante de $16 \%$ al año (decrecen si sólo se toman en cuenta los precios); fertilizantes al $7 \%$; electricidad a casi $8 \%$; etc.

Los coeficientes de insumos se incrementan como resultado de los precios en todos los sectores (lo que probablemente lleve a valores agregados menores); su incremento ocurre también al nivel de coeficientes observados. En los sectores "tradicionales", hay un incremento ex-ante, mientras que aquellos identificados como "modernos" muestran decrementos. Probablemente ésto pueda reforzar la conjetura de "productividad" mencionada anteriormente.

Se puede también pensar que la presencia de lo que se ha estado manejando como cambio $e x$-ante puede ser interpretada como evidencia de que la elasticidad de sustitución no es igual a uno. Esto es materia de investigación ulterior.

VIII. UN MODELO, LIBRE DE FUNCIÓN PRODUCCIÓN, DEL CAMBIO TECNOLÓGICO

Sean $x_{j}$ el producto bruto del sector $j, x_{i}$ el flujo de insumo $i$ en el sector $j$. Sabemos que el coeficiente monetario $p_{i} x_{i j} / p_{j} x_{j}$ no es sino el exponente de una función producción Cobb-Douglas, si esta es la imagen de una tecnología ex-ante. Si la tecnología ex-ante obedece la CES, los coeficientes físicos están dados por:

$$
a_{i j}=x_{i j} / x_{j}=\left(\frac{p_{i}}{p_{j} \theta_{i j}}\right)^{-\sigma} j
$$

en que

$$
x_{j}=\left(\Sigma_{i} \theta_{i j} x_{i j}{ }^{-\rho_{j}}\right)^{-1 / \rho_{j}} \text { y } \rho_{j}=\left(1-\sigma_{\mathrm{j}}\right) / \sigma_{\mathrm{j}}
$$

Supóngase elasticidades de sustitución $\sigma_{i k j}$, del insumo $i$ por el insumo $k$ en el sector $j$, no necesariamente iguales, como en la CES, pero al menos localmente constantes; véase Allen [1938].

$$
\sigma_{i k j}=-\Delta \log \left(x_{i j} / x_{k j}\right) / \Delta \log \left(p_{i} / p_{k}\right)
$$

Sean, para $j$ fija $\delta$ el vector de elementos $\Delta \log p_{i}, \xi_{j}$ el vector de elementos $\Delta \log x_{i j}$, y $S_{j}$ la matriz de elementos $\sigma_{i k j}$. Sea, en general, $\hat{v}$ la matriz diagonal construida del vector $v$ y $\imath$ un vector de unidades. Entonces (8.1) se puede escribir como:

$$
S_{j} \hat{\delta}-\delta S_{j}=\xi_{j} \iota^{\prime}+\iota \xi_{j}
$$


ecuación que, en principio, permite la determinación de $\xi_{j}$. Si se aproxima el lado derecho al lado izquierdo de (5.2) por mínimos cuadrados, con $\iota^{\prime} \xi_{j}=0$, se obtiene: ${ }^{9}$

$$
\xi_{j}=\frac{1}{m}\left(S_{j} \delta-\hat{\delta} S_{j}\right)
$$

en que $m$ es el número de insumos. Re-escribiendo (5.3) se ve que:

$$
\begin{gathered}
\Delta \log x_{i j}=\frac{1}{m}\left(\Sigma_{k}{ }_{i k j} \Delta \log p_{k}-\Delta \log p_{i} \Sigma_{k}{ }_{i k j}\right) \\
=\mu_{i j} \xi_{j}\left\{\Sigma_{k} w_{i k j} \Delta \log p_{k}-\Delta \log p_{i}\right\}
\end{gathered}
$$

El primer término dentre de llaves en la última expresión en (5.4) es una media ponderada de cambios logarítmicos en los precios, con ponderaciones proporcionales a las elasticidades de sustitución ex-post (8.1). Puede verse como el cambio logarítmico de un índice de precios:

$$
\Delta \log \pi_{i j}=\Sigma_{k} w_{i k j} \log p_{k}
$$

Ahora bien, $\mu_{i j}$ se puede evaluar como sigue:

$$
\begin{array}{r}
\mu_{i j}=\frac{1}{m} \Sigma_{k} \sigma_{i k j}=\frac{\mathrm{I}}{m} S_{j \iota} \\
=\frac{1}{m} \hat{\delta}^{-1}\left(S_{j} \hat{\delta}-\xi_{j} \iota^{\prime}+\iota \xi_{j}^{\prime}\right) \iota
\end{array}
$$

utilizando (8.2). Esto es igual a:

$$
\frac{1}{m} S_{j \iota}=\frac{1}{m} \hat{\boldsymbol{\delta}}^{-1} S_{j} \delta-\hat{\boldsymbol{\delta}}^{-1} \xi_{j}
$$

ya que $l^{\prime} \xi_{j}=0$. El primer término de arriba es:

$$
\frac{1}{m} \Sigma_{k} \sigma_{i k j}\left(\Delta \log p_{k} / \Delta \log p_{i}\right)=\mu_{i j} \Delta \log \pi_{\pi_{j}} / \Delta \log p_{j}
$$

el segundo es $\Delta \log x_{i} / \Delta \log p_{i}$. Entonces:

$$
\mu_{i j}\left(1-\Delta \log \pi_{i j} / \Delta \log p_{i}\right)=\Delta \log x_{i j} / \Delta \log p_{i}
$$

$\mu_{i j}$ es una modificación simple de la elasticidad-precio de $x_{i j} ; s i \Delta \log _{i j} /$ $\Delta \log p_{i}=0$ (como en ćualquier modelo de equilibrio parcial) $\mu_{i j}$ es la elasticidad-precio directa de $x_{i j}$.

Todo ésto sugiere ecuaciones de demanda del tipo Barten-Theil para $x_{i j}$ :

$$
\begin{gathered}
\Delta \log x_{i j}=\mu_{i j} \Delta \log \left(p_{i} / \pi_{i j}\right) \\
=\mu_{i j} \Sigma_{k} C_{i k j} \Delta \log p_{k}
\end{gathered}
$$

${ }^{9}$ Minimicese $\operatorname{tr}\left(S_{j} \hat{\delta}-\hat{\delta} S_{j}-\xi_{j} \iota^{\prime}+\imath \xi_{j}\right)^{\prime} \quad\left(S_{j} \hat{\delta}-\hat{\delta} S_{j}-\xi_{j} \iota^{\prime}+\imath^{\prime} \xi_{j}^{\prime}\right) \operatorname{con} \iota \xi_{j}=0$. 
El cambio tecnológico ex-ante estaría medido por cambios en los parámetros de la ecuación (8.6). Esto requeriría una muestra muy grande; se ha sugerido utilizar ecuaciones del tipo:

$$
\Delta \log x_{i j}=C_{i o j}+\Sigma_{k i} C_{i k j} \Delta \log p_{k}
$$

en que $C_{i o j}$ es una tasa de cambio de $x_{i j}$ en el tiempo, que se tomará como debida a cambio tecnológico, y $C_{i k j}$ es proporcional a la elasticidad de sustitución promedio de largo plazo. Se están llevando a cabo investigaciones empíricas sobre las ecuaciones (8.6) y (8.7).

\section{REFERENCIAS}

R.G.D. Allen, Mathematical Analysis for Economists, Londres, Macmillan, 1938.

M. Bacharach, Biproportional Matrices and Input-Output Change, Cambridge University Press, 1970.

W.E. Deming, y F.F. Stephan, "On a Least Squares Adjustment of a Sampled Frequency Table when the Expected Marginal Totals are Known", Annals of Mathematical Statistics, 11 (1940) pp. 427-444.

D. Friedlander, "A Technique for Estimating a Contingence Table, given the Marginal Totals and some Supplementary Data", Journal of the Royal Statistical Society, Series A, 124 (1961), pp. 412-420.

L.R. Klein, "On the Interpretation of Professor Leontief's System". Review of Economic Studies, 19 (1952-1953).

T.C. Koopmans (Comp.), Activity Analysis of Production and Allocation, Nueva York, Wiley \& Sons, 1951.

T.C., Matuszewski, P.R. Pitts y J.A. Sawyer, "Linear Programming Estimates of Changes in Input Coefficients", Canadian Journal of Economics and Political Science, 30 (1964), pp. 203-210.

M. Morishima y Y. Murata, "An Input-Output Analysis of Disguised Unemployment in Japan, 1951-1965". En: Morishima et al., pp. 243-300.

Morishima, et al., The Working of Econometric Models, Cambridge University Press, 1972.

H.A. Simon, "Effects of Technological Change in a Linear Model". En Koopmans, pp. $260-277$.

M. Satto, "A General Equilibrium Analysis of Prices and Outputs in Japan, 19531965". En Morishima et al., pp. 147-240.

H. Theil, Applied Economic Forecasting, Amsterdam, North Holland, 1966.

R. Stone, et al. (Comp.), A Computable Model of Economic Growth. Londres, Chapman and Hall, Ltd., varias fechas (en especial Vol. 3, 1953).

C.B. Tilanus, Input-Output Experiments; the Netherlands. 1948-1961, Rotterdam, Universitaire Press, 1966

C.B. Tilanus, y R. Harkema, "Input-Output Predictions of Primary Demand. The Netherlands, 1948-1958. Documento 6264, Econometric Institute, Rotterdam, 1962.

C.B. Tilanus y G. Rey, "Input-Output Forecasts for the Netherlands, 1949-1958". Econometrica, 31 (1963), pp. 454-453.

G. Rev. y C.B. Tilanus, "Input-Output Volume and Value Predictions for the Netherlands, 1948-1958". International Economic Review, 5 (1964), pp. 34-45.

P., Uribe, C.G. de Leeuw y H. Theil, "The Information Approach to the Prediction of International Trade Flows", Review of Economic Studies 33 (1966), pp. 209-219.

H. Uzawa, "A Generalization of the CES Production Function". Review of Economic Studies, 29, (1962), pp. 126-152. 